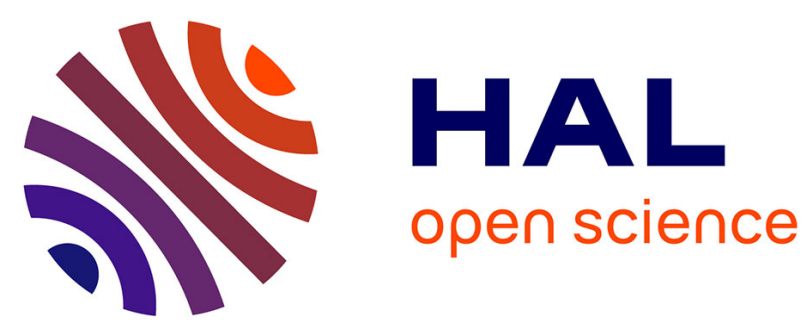

\title{
Mise en oeuvre de la méthode flash pour la mesure de diffusivité thermique sur des matériaux liquides ou fondus en fonction de la température. Application aux polymères
}

\author{
C. Gobbé, J. Gounot, M. Bazin
}

\section{To cite this version:}

C. Gobbé, J. Gounot, M. Bazin. Mise en oeuvre de la méthode flash pour la mesure de diffusivité thermique sur des matériaux liquides ou fondus en fonction de la température. Application aux polymères. Revue de Physique Appliquée, 1989, 24 (12), pp.1119-1128. 10.1051/rphysap:0198900240120111900 . jpa-00246149

HAL Id: jpa-00246149

https://hal.science/jpa-00246149

Submitted on 1 Jan 1989

HAL is a multi-disciplinary open access archive for the deposit and dissemination of scientific research documents, whether they are published or not. The documents may come from teaching and research institutions in France or abroad, or from public or private research centers.
L'archive ouverte pluridisciplinaire HAL, est destinée au dépôt et à la diffusion de documents scientifiques de niveau recherche, publiés ou non, émanant des établissements d'enseignement et de recherche français ou étrangers, des laboratoires publics ou privés. 
Classification

Physics Abstracts

$44.10-44.50-66.60-66.70-07.20$

\title{
Mise en cuvre de la méthode flash pour la mesure de diffusivité thermique sur des matériaux liquides ou fondus en fonction de la température. Application aux polymères
}

\author{
C. Gobbé, J. Gounot et M. Bazin \\ L.E.P.T.-ENSAM, URA CNRS 873, 33405 Talence Cedex, France
}

(Reçu le 22 juin 1989, révisé le 4 septembre 1989, accepté le 8 septembre 1989)

\begin{abstract}
Résumé. - Une mise en œuvre de la méthode flash appliquée à la détermination de la diffusivité thermique dé matériaux solides ou liquides entre $20^{\circ} \mathrm{C}$ et $300{ }^{\circ} \mathrm{C}$ est présentée. L'influence de la présence du creuset nécessaire à ce type de mesures est étudiée. Nous en déduisons une procédure spécifique d'identification de la diffusivité, procédure au cours de laquelle une évaluation de la chaleur volumique est fournie. Une description d'ensemble du dispositif expérimental est proposée et la comparaison de quelques mesures avec les valeurs courantes de la littérature contribue à la validation de notre démarche théorique et expérimentale.
\end{abstract}

\begin{abstract}
The flash method is applied to determine solid and liquid thermal diffusivity on temperature range of $20^{\circ} \mathrm{C}-300^{\circ} \mathrm{C}$. The influence of the needed vessel used for liquid confinment is studied. We deduce a specific process for the diffusivity identification and an estimation of the heat capacity. The experimental apparatus is described. The experimental and theoretical approach is validated by a comparison between our measurements and available results in the literature.
\end{abstract}

Depuis 1985, nous avons entrepris, à l'instigation du centre de recherche de la Société ATOCHEM (CERDATO, 27470 Serquigy, France) et avec son concours financier, la mise au point de dispositifs de mesures destinés à la caractérisation thermophysique des polymères, indispensable à la modélisation par C.A.O. du moulage des matières plastiques. Cette collaboration a abouti en 1986 à la réalisation d'un conductivimètre haute pression et moyenne température [1]. Nous proposons aujourd'hui un dispositif de mesure de diffusivité thermique par méthode flash en fonction de la température $\left(20^{\circ} \mathrm{C}-300^{\circ} \mathrm{C}\right)$ sur des polymères pouvant être à l'état solide ou fondu.

Le choix vers lequel nous nous sommes orientés, à savoir la méthode flash, est directement lié au fait qu'il s'agit d'une méthode rapide et fiable si tant est que la technique d'identification de la diffusivité ne soit pas trop restrictive ou approximative.

Rappelons que le principe de la méthode consiste à soumettre un système à une perturbation thermique de courte durée (flash) et à observer la réponse en température qu'elle engendre. Le système étudié peut être un échantillon à faces parallèles soumis à une impulsion thermique sur l'une de ses faces, l'évolution de la température étant enregistrée sur la face opposée [2-6]. Il peut s'agir aussi d'un échantillon à géométrie cylindrique soumis à une impulsion axiale, la température étant relevée sur une génératrice externe du cylindre [7, 8]. On peut noter, par ailleurs, que jusqu'à présent les principales applications de la méthode flash ont été limitées à des matériaux indéformables bien que quelques essais ponctuels aient été menés sur des milieux liquides [68] ou plus récemment des milieux non consolidés [9].

Dans tous les cas, la précision avec laquelle la diffusivité thermique est déduite du thermogramme dépend du choix des conditions aux limites relatives aux échanges du système avec le milieu extérieur dans la modélisation du processus. Depuis le modèle adiabatique proposé par Parker [2], de nombreux auteurs ont tenté d'affiner la modélisation en présentant plusieurs méthodes d'estimation des échanges avec le milieu extérieur [3]. L'originalité de la démarche de Degiovanni [10], dont nous nous sommes inspirés, réside dans le fait que le principe d'identification qu'il propose n'introduit aucune hypothèse restrictive quant à ces échanges. Sa techni- 
que de dépouillement du thermogramme les prend en compte sans qu'il soit nécessaire de les évaluer explicitement.

La caractérisation de liquides s'avère plus complexe que celle de milieux indéformables tant du point de vue technologique que du point de vue modélisation compte tenu de la nécessité d'adjoindre un contenant. Dans un premier temps, le cahier des charges relatif à la mesure de diffusivité sur des liquides ou polymères fondus a donc été défini. Ce travail a abouti à la réalisation d'un dispositif expérimental dont les principaux aspects sont décrits ici. Dans un second temps, une procédure de dépouillement des thermogrammes relatifs au système défini et applicable dans de nombreux cas est proposée. Précisons que cette procédure, applicable comme nous le verrons à de nombreux systèmes stratifiés, fournit une évaluation de la chaleur volumique des échantillons.

Les premières mesures effectuées sur quelques matériaux tests sont présentées et comparées aux valeurs de la littérature $[12,13]$.

\section{Dispositif expérimental.}

1.1 DÉFINITION DU SYSTÈME. - Le principe expérimental de la méthode flash sur un échantillon cylindrique à faces parallèles a été présenté de façon suffisamment exhaustive dans la littérature. Précisons cependant trois éléments indispensables à la réalisation d'une mesure pour mettre en évidence les contraintes liées à la mise en place d'une solution technique sur des échantillons liquides :

- l'absorption du rayonnement sur la face éclairée par le flash doit être instantanée et uniforme ;

- les transferts au sein de l'échantillon doivent être exclusivement de type diffusif ;

- la mesure de la réponse en température, effectuée dans notre cas par des détecteurs semiconducteurs à contacts séparés, de grande sensibilité et recommandés dans ce type d'applications [14], nécessite la présence d'une liaison métallique locale.

D'autres impératifs, plus spécifiquement liés à la présence du creuset contenant l'échantillon doivent être respectés :

- les surfaces latérales du creuset doivent être constituées d'un matériau peu conducteur afin de limiter les fuites thermiques radiales et la conduction longitudinale ;

- pour éviter des problèmes d'étanchéité essentiellement liés aux dilatations différentielles susceptibles d'apparaître lors de la montée en température, le fond du creuset et ses parois latérales doivent être usinés d'un seul bloc.

Pour répondre à l'ensemble de ces exigences, nous proposons le système suivant (Fig. 1). L'échantillon

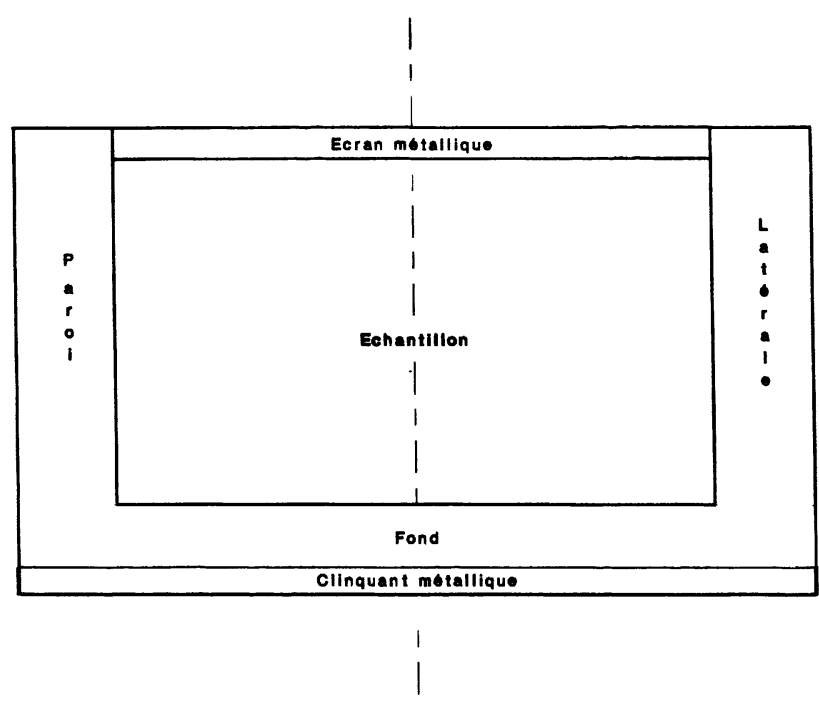

Fig. 1. - Schéma du système soumis au flash.

[Schematic representation of the system lighted by the flash tube.]

est placé dans un creuset de polyimide (polymère relativement isolant : $\lambda=0,35 \mathrm{~W} / \mathrm{m}{ }^{\circ} \mathrm{C}, a=2.8 \times$ $10^{-7} \mathrm{~m}^{2} / \mathrm{s}$ ) dont la tenue mécanique en température est assurée jusqu'à $300^{\circ} \mathrm{C}$. Ce creuset a pour dimensions : $35 \mathrm{~mm}$ de diamètre intérieur, $37 \mathrm{~mm}$ de diamètre extérieur, $18,5 \mathrm{~mm}$ de hauteur, l'épaisseur du fond étant de $1 \mathrm{~mm}$. Un écran opaque est placé sur l'échantillon afin d'éviter que, pour des milieux transparents, le transfert ne s'effectue par rayonnement au sein de l'échantillon. Pour obtenir une bonne homogénéité du flux absorbé, cet écran est constitué par un clinquant d'acier doux (épaisseur $1 / 10 \mathrm{~mm}$ ) dont la surface exposée au flash est noircie pour améliorer l'absorption du rayonnement. Le contact électrique nécessaire à la mesure de température sous le creuset est assuré par un second clinquant métallique identique au premier, collé au creuset pour maintenir un bon contact thermique.

1.2 DESCRIPTION D'ENSEMBLE DU DISPOSITIF. L'ensemble de l'appareil (Fig. 2) se présente sous la forme d'une cellule cylindrique en acier inoxydable (66) dont le couvercle (68) est mobile autour de l'axe (43). Ce couvercle est équipé du flash (6) et du capteur de proximité (37) destiné à la mesure de l'épaisseur de l'échantillon. De plus, une ouverture est prévue pour la mise en place de l'échantillon. Le couvercle, ainsi conçu, peut être déplacé, par simple rotation, dans la position adéquate à la phase d'expérimentation en cours. Des plots de positionnement sont prévus à cet effet.

L'enceinte contient essentiellement le four, le volume restant étant comblé par de la vermiculite pour en assurer l'isolation. Les parois du four sont usinées dans un rond de céramique (24) autour duquel est bobinée une résistance chauffante. Ther- 


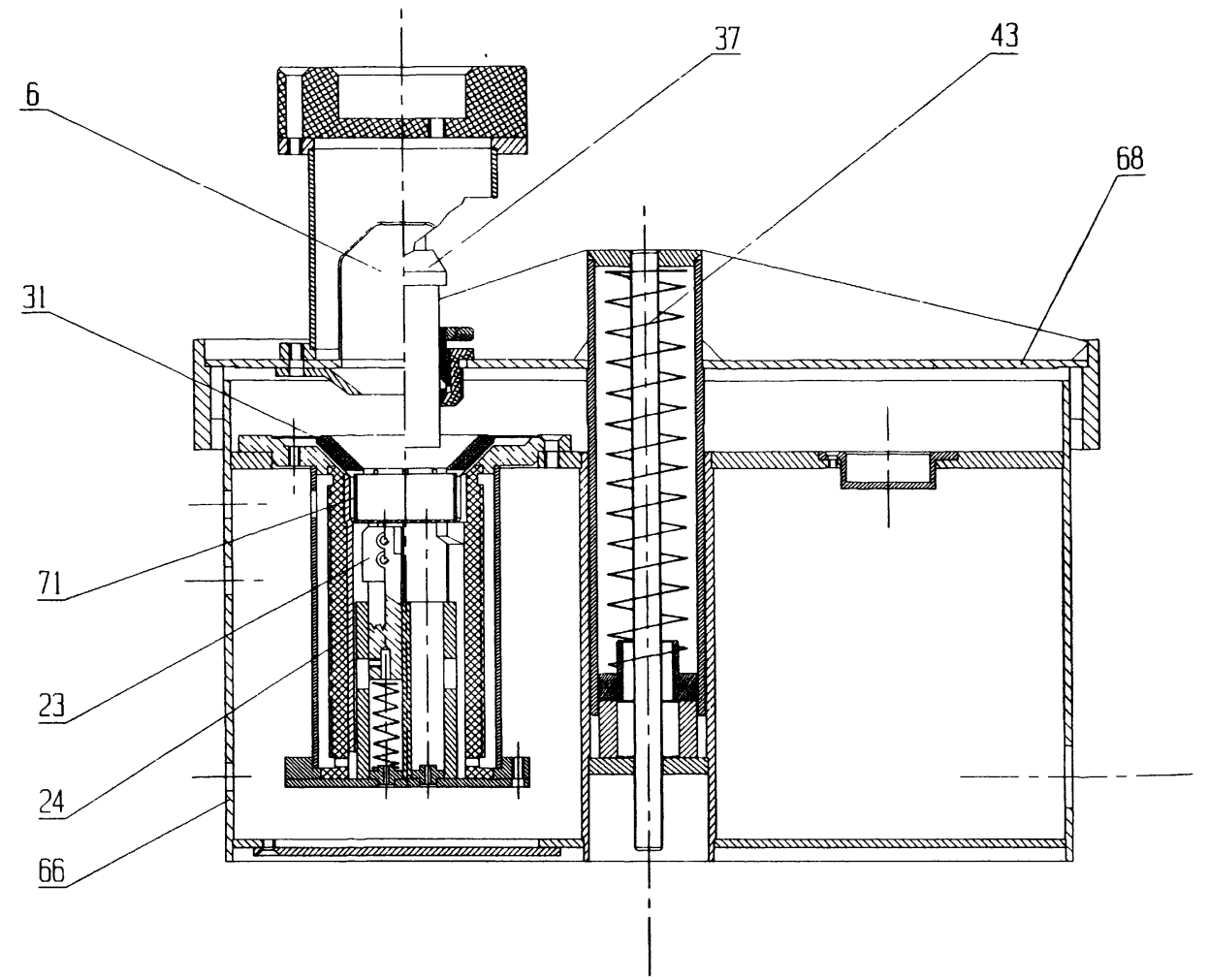

Fig. 2. - Dispositif expérimental.

[Experimental apparatus.]

mocoax de $60 \Omega$. Le tout est placé dans une chemise métallique et fixé à demeure dans l'enceinte. Un second chemisage métallique à l'intérieur du four a été mis en place pour limiter le gradient thermique longitudinal.

Dans sa partie haute, le four présente un rebord circulaire pour reposer le creuset cylindrique dans lequel est placé l'échantillon. Un diaphragme réfléchissant sur sa face supérieure (31) assure le centrage $\mathrm{du}$ creuset et évite l'absorption de lumière parasite par d'autres éléments que l'échantillon. La température du four est contrôlée par un thermocouple de type $\mathrm{K}$ relié à une régulation PID (Eurotherm $818 \mathrm{~S}$ ).

1.3 LES PRINCIPAUX ÉLÉMENTS DE MESURE. Pour mesurer l'épaisseur de l'échantillon, on dispose d'un capteur de proximité de type capacitif (Capacitec-Proximic 3101SP). Le capteur fournit un signal proportionnel à la distance entre sa partie sensible et une cible conductrice reliée à la masse du système. Dans notre dispositif, la cible détectée est le clinquant supérieur de l'échantillon, la masse électrique étant reliée à l'enceinte. Une réponse linéaire spécifique à ce montage a été mise en évidence. L'épaisseur de l'échantillon est déduite des distances du capteur à l'échantillon et au plan de référence (plan où repose le récipient). On estime que l'erreur absolue commise sur cette valeur est de $\pm 5 / 100 \mathrm{~mm}$.
La mesure de température sous le creuset se fait à l'aide de deux détecteurs (23) semi-conducteurs dopés respectivement positivement et négativement. Ces détecteurs sont en contact ponctuel avec le clinquant placé sous le fond du creuset (71), leur autre extrémité étant maintenue dans des blocs de cuivre isothermes.

\section{Modélisation.}

2.1 ModÉlisATION BIDIMENSIONNELlE. - La détermination de la diffusivité thermique à partir d'un thermogramme expérimental est effectuée par comparaison avec un thermogramme calculé numériquement à partir d'un jeu de données parfaitement définies. Cette comparaison est effectuée, soit sur quelques temps caractéristiques [10], déterminés par l'amplitude du signal rapportée à l'amplitude maximale (temps correspondant à $1 / 3,1 / 2,2 / 3$ ou $5 / 6$ du signal maximal par exemple, notés respectivement $\left.t_{1 / 3}, t_{1 / 2}, t_{2 / 3}, t_{5 / 6}\right)$, soit sur l'ensemble du thermogramme [11]. Si l'identification des deux thermogrammes est possible, alors les propriétés du milieu étudié sont assimilables aux valeurs des paramètres indépendants introduits dans le calcul.

La détermination théorique de l'évolution de la température en fonction du temps sur la face arrière 
de l'échantillon nécessite une modélisation a priori bidimensionnelle prenant en compte la symétrie cylindrique du système échantillon + creuset + clinquants. Dans le cas très général d'un échantillon homogène soumis à une impulsion thermique de courte durée, le transfert de chaleur en régime transitoire est décrit par l'équation :

$$
\frac{1}{a} \frac{\partial T}{\partial t}=\frac{\partial^{2} T}{\partial r^{2}}+\frac{1}{r} \frac{\partial T}{\partial r}+\frac{\partial^{2} T}{\partial z^{2}}
$$

avec pour conditions aux limites :

$$
\begin{aligned}
& \left.\frac{\partial T}{\partial z}\right|_{z=0}=\frac{h_{0}}{\lambda}\left(T(r, 0, t)-T_{\infty}\right) \quad \forall r, t \\
& \left.\frac{\partial T}{\partial z}\right|_{z=e}=-\frac{h_{\mathrm{e}}}{\lambda}\left(T(r, e, t)-T_{\infty}\right) \quad \forall r, t \\
& \left.\frac{\partial T}{\partial r}\right|_{r=R}=-\frac{h_{\mathrm{R}}}{\lambda}\left(T(R, z, t)-T_{\infty}\right) \quad \forall z, t
\end{aligned}
$$

et pour condition initiale :

$$
\begin{array}{lll}
T(r, z, 0)-T_{\infty}=\frac{Q}{\rho C \varepsilon} & \text { si } & 0 \leqslant z<\varepsilon \\
T(r, z, 0)-T_{\infty}=0 & \text { si } & z>\varepsilon
\end{array}
$$

où $\varepsilon$ représente l'épaisseur $a$ priori très faible sur laquelle il y a absorption instantanée de l'énergie émise par le flash.

Etant donné l'hétérogénéité du système à modéliser une méthode de discrétisation de l'équation (1) par volumes finis a été choisie et l'intégration temporelle du système obtenu a été effectuée par une méthode A.D.I. (Alternating Direction Implicit). Le détail de cette modélisation n'est pas présentée ici mais seulement les résultats qui en sont issus, puisqu'elle ne constitue qu'une étape nécessaire à la justification de l'emploi d'un modèle unidimensionnel lors du dépouillement des thermogrammes. Notons qu'il apparaît neuf paramètres adimensionnels dans l'écriture du système pour une structure quadricouche, à savoir les chaleurs volumiques et les conductivités des différentes couches rapportées à celles de l'échantillon ainsi que les coefficients d'échange $h_{0}, h_{e}$ et $h_{R}$ intervenant sous la forme de nombres de Biot. Dans le calcul, les variations de diffusivité et conductivité du creuset et des clinquants en fonction de la température sont prises en compte. Les valeurs de $h_{0}, h_{e}$ et $h_{\mathrm{R}}$ ont été supposées comprises entre 0 et $25 \mathrm{~W} / \mathrm{m}^{2}{ }^{\circ} \mathrm{C}$ et l'épaisseur de l'échantillon entre $8 \mathrm{~mm}$ et $14 \mathrm{~mm}$.

Le calcul de $T(e, r, t)$ s'avère performant quant aux résultats obtenus mais demeure assez long d'exécution. Par ailleurs, il ne permet pas, étant donné le nombre conséquent de paramètres, d'aboutir à une identification simple de la diffusivité quel que soit le type de configuration. La comparaison des résultats obtenus à partir de la modélisation bidimensionnelle d'un échantillon homogène d'une part, d'un échantillon placé dans un creuset d'autre part, et ce, dans tous les cas envisagés plus haut, montre que l'influence de la présence du récipient sur la valeur des temps caractéristiques du thermogramme peut atteindre $20 \%$. Un calcul itératif a donc été envisagé pour l'identification de la diffusivité thermique. Pour conserver à la méthode flash sa simplicité, sa rapidité et une possibilité d'implantation du dépouillement sur micro-ordinateur, la détermination de $a$ à partir d'un calcul itératif 2D n'était pas envisageable. Cependant, la comparaison des résultats du calcul $2 \mathrm{D}$ avec ceux d'une modélisation 1D par la méthode des quadripôles a permis de définir les critères à respecter pour que la réponse enregistrée par les capteurs de température puisse être interprétée à l'aide d'une méthode de dépouillement supposant un transfert unidirectionnel.

2.2 MODÉliSATION 1D PAR LA MÉTHODE DES QUADRIPÓLES. - Il s'agit d'une méthode de type « analogie électrique » déjà présentée dans la littérature [15], qui consiste à considérer le milieu comme un quadripôle soumis à un signal d'entrée connu et dont on calcule le signal de sortie. La résolution est simplifiée par l'utilisation de la transformation de Laplace.

Le multicouche, sans résistance de contact, composé de « l'empilement » du clinquant supérieur, de l'échantillon, du fond du creuset et du clinquant arrière (Fig. 3a) se comporte comme 4 quadripôles

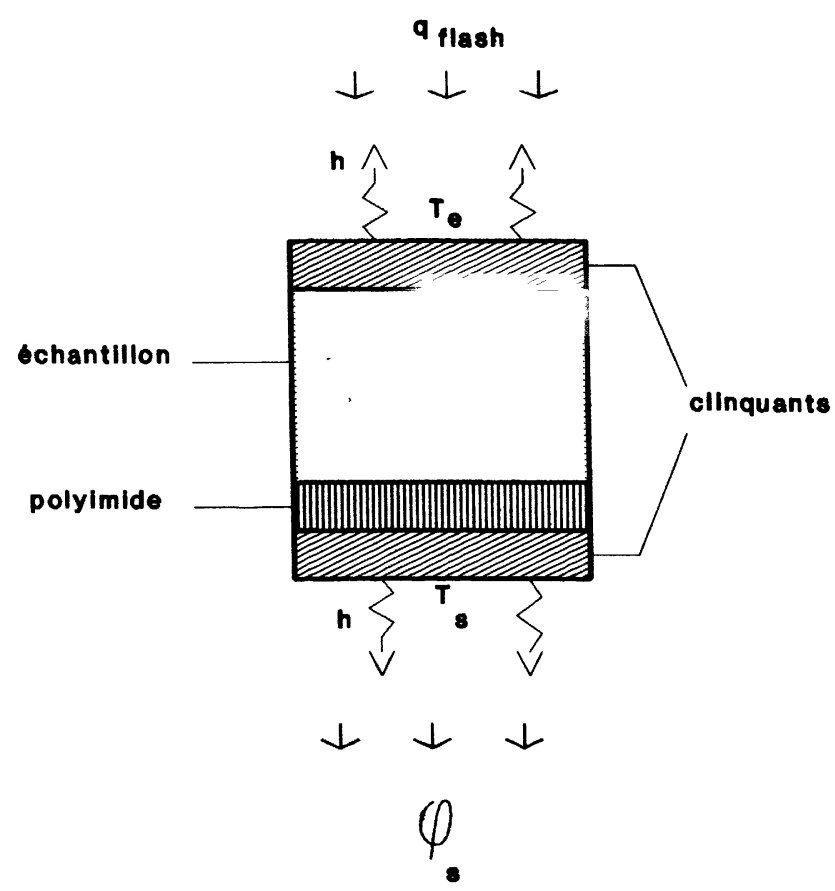

Fig. 3a. - Quadricouche avec échanges convectifs sur les deux faces parallèles.

[Fourlayer system with convective boundary conditions on both parallel sides.] 


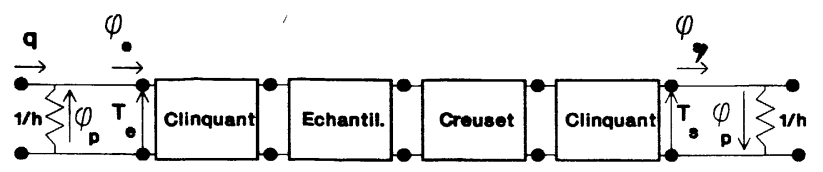

Fig. 3b. - Quadripôle équivalent à la figure $3 a$.

[Equivalent quadripole corresponding to the system figure 3a.]

placés en série comme le montre la figure $3 \mathrm{~b}$. A chaque quadripôle est associée une matrice de transfert. Le signal à l'entrée du multicouche est lié au signal de sortie par le produit des matrices de transfert relatives à chaque milieu

$$
\left[\begin{array}{l}
\theta_{\mathrm{e}} \\
\varphi_{\mathrm{c}}
\end{array}\right]=\tau_{1} \tau_{2} \tau_{3} \tau_{1}\left[\begin{array}{l}
\theta_{\mathrm{s}} \\
\varphi_{\mathrm{s}}
\end{array}\right]=\left[\begin{array}{ll}
A & B \\
C & D
\end{array}\right]\left[\begin{array}{l}
\theta_{\mathrm{s}} \\
\varphi_{\mathrm{s}}
\end{array}\right]
$$

$\tau_{1}$ : matrice relative au clinquant,

$\tau_{2}$ : matrice relative à l'échantillon,

$\tau_{3}$ : matrice relative au creuset.

Les matrices $\tau_{i}$ ne sont parfaitement définies que si l'on connaît les valeurs de $\lambda$ et $a$ pour chaque milieu ce qui revient encore à définir les valeurs de $\rho C$ et $a$.

La condition à la limite mixte, caractérisée par un coefficient d'échange $h$, est traduite dans le cadre de l'analogie électrique par la présence d'un quadripôle particulier, constitué d'une résistance $1 / h$ placée entre les bornes d'entrée et celles de sortie.

Dans ce type de représentation, on obtient :

$$
\begin{aligned}
& \varphi_{\mathrm{e}}=q-h \theta_{\mathrm{e}} \\
& \varphi_{\mathrm{s}}=h \theta_{\mathrm{s}}
\end{aligned}
$$

où $q$ est la transformée de Laplace du flux émis par le flash et parvenant à l'échantillon, soit en tenant compte de la relation (7):

$$
\theta_{\mathrm{s}}=\frac{q}{h A+h^{2} B+C+h D}
$$

En prenant la transformée inverse de Laplace de $\theta_{\mathrm{s}}$, on obtient l'évolution de la température $T_{\mathrm{s}}$ sur la face arrière du multicouche à chaque instant.

\subsection{CRITÈres DE VALIDITÉ DE LA MODÉlisATION} 1D. - Nous avons illustré le fait que, dans certains cas, l'aspect bidimensionnel du transfert thermique pouvait être négligé en comparant les thermogrammes calculés en coordonnées réduites, d'une part à l'aide de la modélisation bidimensionnelle et d'autre part à l'aide du calcul monodimensionnel (Figs. 4a et 4b).

Dans le premier exemple, il y a concordance parfaite entre toutes les courbes ce qui tend à prouver qu'un modèle $1 \mathrm{D}$ est suffisant dans ce type de configuration. Par contre, dans le second cas, on

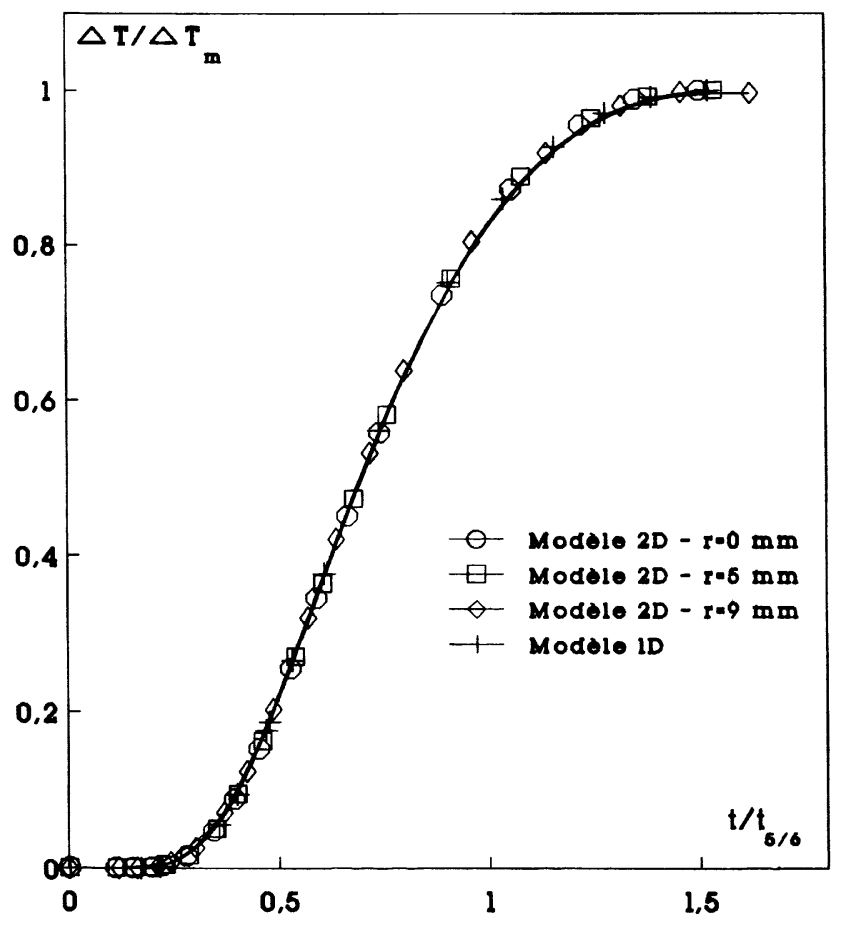

a)

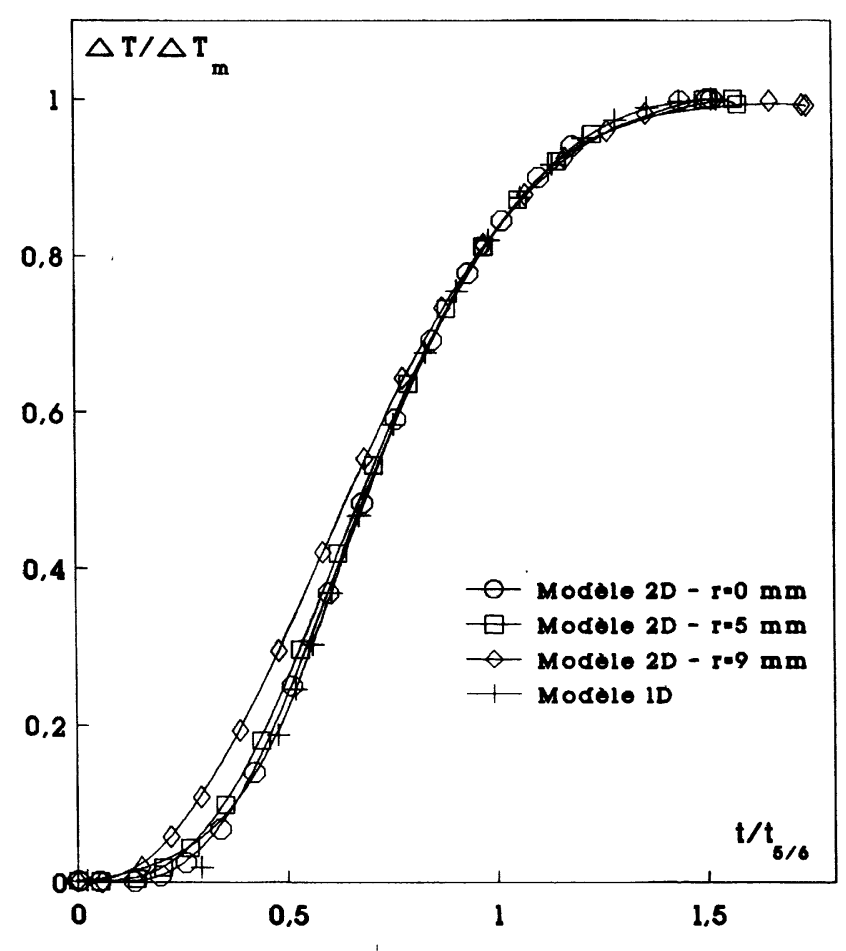

b)

Fig. 4. - Comparaison des thermogrammes $1 \mathrm{D}$ et 2D calculés : a) effet bidimensionnel négligeable $\left(a_{3} / a_{2}=\right.$ 7, $\left.\lambda_{3} / \lambda_{2}=3\right)$, b) effet bidimensionnel non négligeable $\left(a_{3} / a_{2}=15, \lambda_{3} / \lambda_{2}=8\right)$.

[Comparison between computed 1D and 2D thermograms : a) bidimensional effect negligible $\left(a_{3} / a_{2}=7\right.$, $\left.\lambda_{3} / \lambda_{2}=3\right)$, b) bidimensional effect non negligible $\left(a_{3} / a_{2}=15, \lambda_{3} / \lambda_{2}=8\right)$.] 
observe une certaine dispersion entre les différents thermogrammes, dispersion qui s'accroît avec $r$, distance du point de mesure par rapport à l'axe. La déformation du champ thermique n'apparaît que pour des valeurs de conductivité et diffusivité du creuset très supérieures à celles de l'échantillon.

Pour quantifier l'importance relative de cette déformation, une méthode consiste à calculer la diffusivité apparente du système par la méthode de Degiovanni [10], en assimilant ainsi le multicouche à un milieu homogène. Si, quel que soit $r$, la valeur de cette diffusivité reste constante ou subit une variation très limitée, on admet que le flux est unidirectionnel.

Il faut noter que le comportement d'un milieu stratifié n'est pas systématiquement identifiable à celui d'un milieu homogène équivalent ce qui peut remettre en cause la détermination même d'une diffusivité apparente. Ici cependant, compte tenu de la faible épaisseur du creuset et des clinquants par rapport à celle de l'échantillon, le thermogramme relatif au multicouche peut être confondu avec celui $\mathrm{du}$ milieu homogène équivalent comme le montre, à titre d'exemple, la figure 5. Les trois corrélations de Degiovanni [10] peuvent donc être appliquées et aboutissent à la définition d'une diffusivité apparente

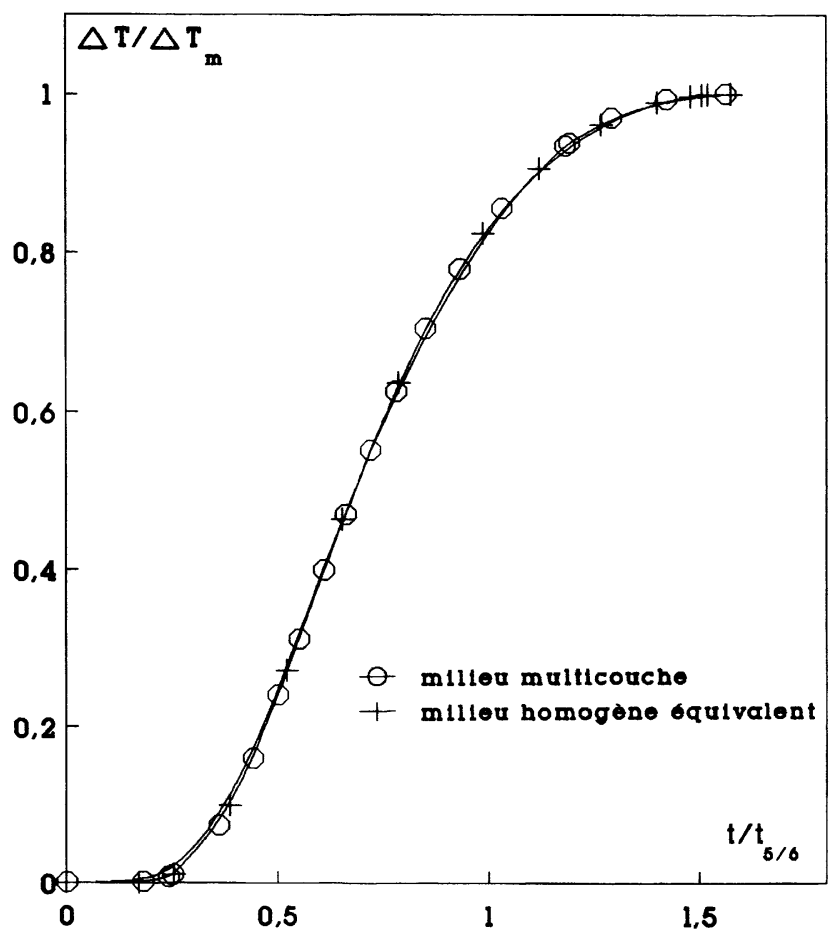

Fig. 5. - Comparaison des thermogrammes calculés pour un milieu multicouche (creuset polyimide, clinquants aciers et $a_{2}=1,63 \times 10^{-7} \mathrm{~m}^{2} / \mathrm{s}$ ) et pour le milieu homogène équivalent $\left(a_{\text {apparent }}=1,68 \times 10^{-7} \mathrm{~m}^{2} / \mathrm{s}\right)$.

[Comparison between computed thermogram related to a multilayer sample (polyimide vessel, steel foils and $\left.a_{2}=1.63 \times 10^{-7} \mathrm{~m}^{2} / \mathrm{s}\right)$ and the equivalent homogeneous medium $\left(a_{\text {apparent }}=1.68 \times 10^{-7} \mathrm{~m}^{2} / \mathrm{s}\right)$.] unique par thermogramme. Ceci constitue la condition sine qua none sans laquelle la procédure d'identification proposée ultérieurement n'est plus applicable.

D'une façon générale, les calculs de $a_{\text {apparent }}$ ont montré que :

- l'influence du rapport des conductivités reste prépondérante comparée à celle du rapport des diffusivités sur la déformation des thermogrammes. Par ailleurs, cette influence est d'autant plus sensible que l'on se situe près des parois ;

- si la conductivité et la diffusivité de l'échantillon sont du même ordre de grandeur ou supérieures à celle du creuset les variations de $a_{\text {apparent }}$ sont nulles sur un rayon de $5 \mathrm{~mm}$ et atteignent $2 \%$ sur un rayon de $9 \mathrm{~mm}$ ce qui s'avère négligeable ;

- dans le cas où le rapport $\lambda_{3} / \lambda_{2}$ atteint une valeur de l'ordre de 3 , la variation de $a_{\text {apparent }}$ reste limitée à $1,5 \%$ si $r \leqslant 5 \mathrm{~mm}$ mais elle peut atteindre pour $r=9 \mathrm{~mm}, 4 \%$ lorsque $a_{3} / a_{2} \leqslant 5$ et $8 \%$ si $5 \leqslant a_{3} / a_{2} \leqslant 7$.

En conclusion, compte tenu des propriétés du creuset, l'aspect bidimensionnel du champ thermique n'est pas sensible pour un rayon inférieur à $5 \mathrm{~mm}$ (soit 2 à 3 fois le rayon effectif de mesure sur le dispositif expérimental) si $\lambda_{2} \geqslant 0,1 \mathrm{~W} / \mathrm{m}^{\circ} \mathrm{C}$ et $a_{2} \geqslant$ $0,4 \times 10^{-7} \mathrm{~m}^{2} / \mathrm{s}$. En deçà de ces limites, la perturbation du champ progresse vers l'axe (Fig. 6) et la détermination même d'une diffusivité apparente devient impossible. La procédure d'identification proposée, basée sur l'hypothèse d'un comportement unidirectionnel, sera donc applicable dans les limites qui viennent d'être précisées.

\section{Procédure d'identification.}

Comme il a déjà été précisé, les coefficients de la matrice qui apparaissent dans la relation (10), et qui sont nécessaires au calcul de $\theta_{s}$ et par conséquent $T_{\mathrm{s}}$ font intervenir les diffusivités respectives des différents milieux (échantillon, creuset et clinquants), leur épaisseur, leur conductivité et le coefficient d'échange $h$ sur les faces de l'échantillon. Les caractéristiques du clinquant et du creuset sont connues, la diffusivité de l'échantillon est, ici, un paramètre d'entrée pour l'identification. Restent à déterminer les valeurs de $h$ et $\lambda_{2}$ ou bien de $h$ et de la chaleur volumique de l'échantillon $\rho_{2} C_{2}$. En fait, ces deux grandeurs peuvent être simplement obtenues à partir du thermogramme expérimental.

3.1 ESTIMATION DE LA CHALEUR VOlUMiQUE. Dans le cas où le système échantillon + creuset + clinquant est adiabatique, l'élévation maximale de température qu'il subit est directement liée à sa 


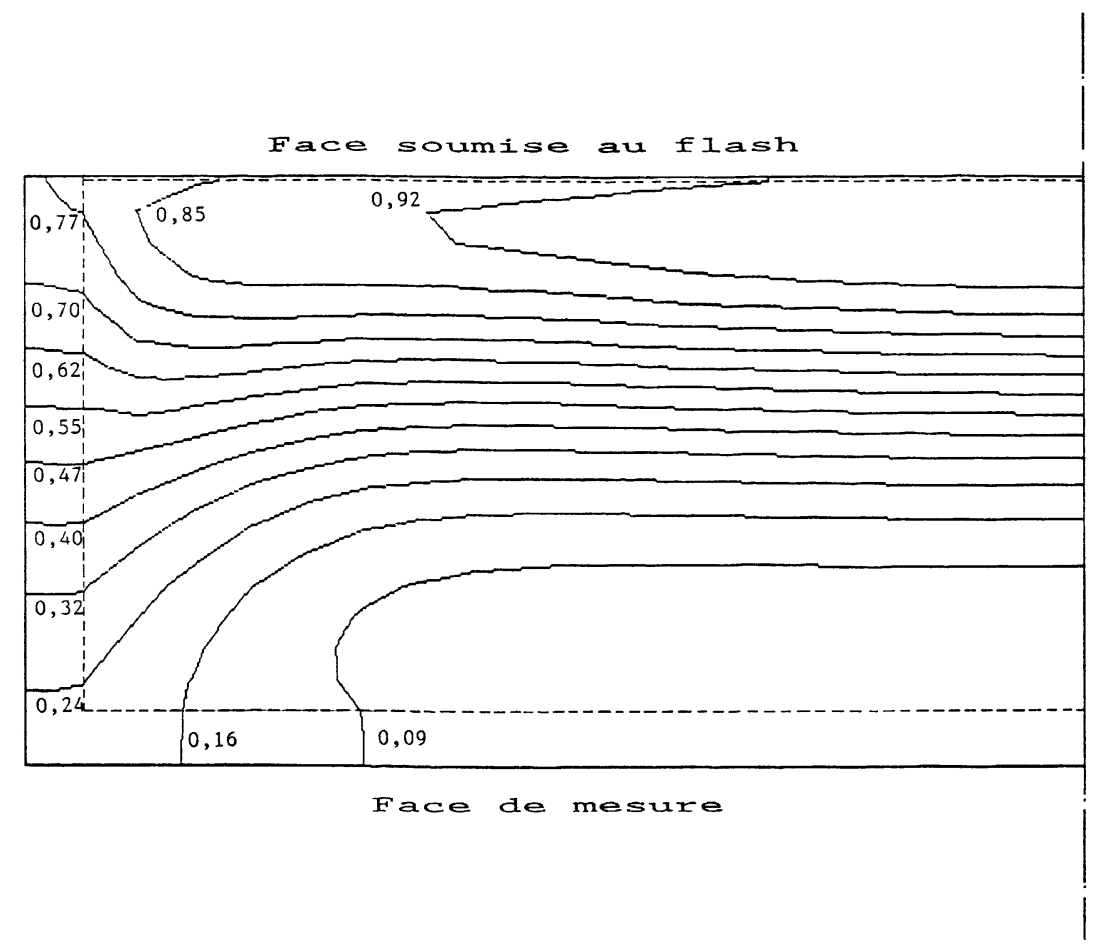

Fig. 6. - Représentation du champ d'isothermes dans l'échantillon au temps $t / t_{5 / 6}=0,33\left(a_{3} / a_{2}=15, \lambda_{3} / \lambda_{2}=8\right)$. [Isothermal field in the sample for $t / t_{5 / 6}=0.33\left(a_{3} / a_{2}=15, \lambda_{3} / \lambda_{2}=8\right)$.]

chaleur volumique par la relation :

$$
\Delta T_{\mathrm{m}}^{(\mathrm{ad})}=\frac{Q}{(\rho C e)_{\text {global }}}
$$

où $Q$ est l'énergie absorbée par unité de surface. Elle peut être évaluée avec une précision suffisante à l'aide d'un échantillon de référence dont on connaît la chaleur volumique. On aura alors :

$$
\begin{array}{r}
(\rho C e)_{\text {global }}=\rho_{2} C_{2} e_{2}+2 \rho_{1} C_{1} e_{1}+\rho_{3} C_{3} e_{3}= \\
=\frac{Q}{\Delta T_{\mathrm{m}}^{(\mathrm{ad})}}
\end{array}
$$

soit :

$$
\rho_{2} C_{2}=\frac{Q}{e \Delta T_{\mathrm{m}}^{(\mathrm{ad})}}-2 \rho_{1} C_{1} \frac{e_{1}}{e}-\rho_{3} C_{3} \frac{e_{3}}{e}
$$

avec $Q=(\rho C e)_{\mathrm{réf}} / \Delta T_{\mathrm{m}_{\mathrm{ref}}}^{(\mathrm{ad})}$.

L'accroissement de température d'un système adiabatique peut être déterminé à l'aide de la simulation par quadripôle dans laquelle on impose $h=0$. Une relation peut être établie entre la valeur obtenue et l'accroissement réel de température [1618]. Ainsi, on a pu vérifier numériquement que, sur l'ensemble des cas envisageables pour des polymères, à savoir $a_{2}$ compris entre $0,4 \times 10^{-7}$ et $2,8 \times$ $10^{-7} \mathrm{~m}^{2} / \mathrm{s}, \lambda_{2}$ compris entre 0,1 et $0,5 \mathrm{~W} / \mathrm{m}{ }^{\circ} \mathrm{C}, h$ compris entre 0 et $50 \mathrm{~W} / \mathrm{m}^{2}{ }^{\circ} \mathrm{C}$ et $e_{2}$ compris entre 8 et $14 \mathrm{~mm}$ :

$$
\Delta T_{\mathrm{m}} / \Delta T_{\mathrm{m}}^{(\mathrm{ad})}=g\left(t_{1 / 2} / t_{5 / 6}\right)
$$

avec $g$ fonction linéaire par morceaux (Fig. 7).

L'accroissement maximum de température $\Delta T_{\mathrm{m}}^{(\mathrm{ad})}$ est donc obtenu directement à partir du thermogramme et par conséquent la chaleur volumique de l'échantillon peut être déduite de la relation (13).

Cette méthode est satisfaisante si :

- la quantité d'énergie $Q$ absorbée par l'échantillon est correctement estimée ;

- la reproductibilité du comportement du flash est bonne.

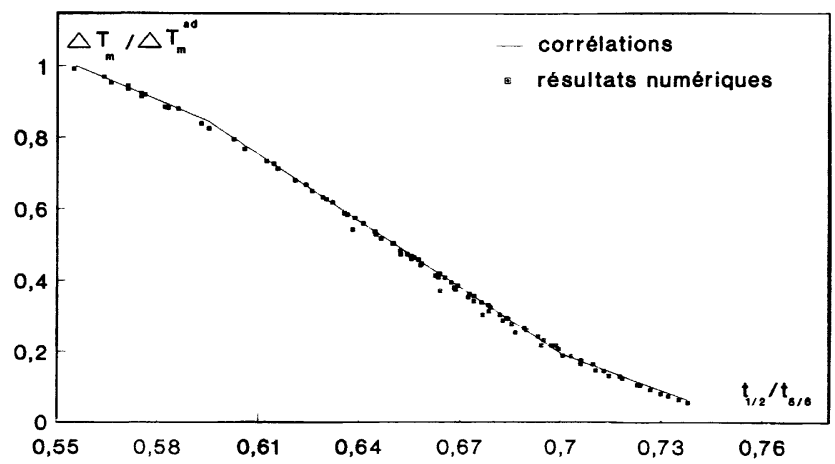

Fig. 7. - Fonction d'identification de $\Delta T_{\mathrm{m}} / \Delta T_{\mathrm{m}}^{(\text {ad })}$.

[Identification function of $\Delta T_{\mathrm{m}} / \Delta T_{\mathrm{m}}^{(\mathrm{ad})}$.] 
On peut supposer que la quantité de chaleur absorbée par l'échantillon, le creuset et les clinquants et l'échantillon de référence est la même pour une même puissance de flash si les surfaces absorbantes ont un comportement identique. Dans ces conditions, on pourrait penser que le fait de noircir la surface de l'échantillon soumise au rayonnement du flash, engendre une bonne reproductibilité du phénomène d'absorption. Ceci serait vrai si l'énergie était, à l'instant initial, répartie sur une surface d'épaisseur infinitésimale pratiquement négligeable. Un certain nombre d'essais, sur différents types de matériaux dont on connaît la chaleur volumique, ont été réalisés pour vérifier la validité de ces hypothèses. Il est apparu que la nature du matériau déterminait directement la quantité d'énergie absorbée par l'échantillon. En d'autres termes, l'épaisseur sur laquelle est absorbé le flux à l'instant initial n'est pas infiniment faible de sorte que les caractéristiques thermophysiques de l'échantillon conditionnent l'absorption du rayonnement incident.

Dans de telles conditions, la méthode flash ne peut être systématiquement étendue à la mesure de chaleur volumique sur des échantillons homogènes dont le comportement serait comparé à celui de l'échantillon de référence. Par contre, dans notre cas de figure, la mesure de la diffusivité s'effectue sur des échantillons liquides ou solides dont la face avant est constituée par un clinquant noirci. Ceci présente un avantage certain quant à la détermination de la chaleur volumique puisque qu'il suffit d'utiliser un échantillon de référence de même nature que les clinquants pour obtenir une absorption équivalente du rayonnement incident.

La quantité d'énergie a été déterminée en fonction de la température de l'essai et de la puissance du flash. Quant à la reproductibilité du flash, elle est estimée à $10 \%$.

\subsection{DÉTERMINATION DU COEFFICIENT D'ÉCHANGE}

$h$. - La forme de la courbe $T(t)$ est directement liée à l'importance des échanges convectifs avec le milieu ambiant. Ainsi le nombre de Biot $\left(\mathrm{Bi}=h e_{2} / \lambda_{2}\right)$ peut lui aussi s'exprimer de façon univoque en fonction de $t_{1 / 2} / t_{5 / 6}$ :

$$
\mathrm{Bi}=f\left(t_{1 / 2} / t_{5 / 6}\right)
$$

avec $f$ fonction semi-logarithmique par morceaux (Fig. 8), les cas envisagés étant les mêmes que ceux précisés au paragraphe précédent. On déduit de la relation précédente une valeur de $h$ pour une valeur fixée de $a_{2}$.

\subsection{PROCÉDURE D'IDENTIFICATION DE LA DIFFUSI-} VITÉ RÉELLE DE L'ÉCHANTILLON. - Etant donné que la présence du récipient et des clinquants d'acier a une influence relativement limitée $(<20 \%)$, la valeur de base de la diffusivité sera la valeur de

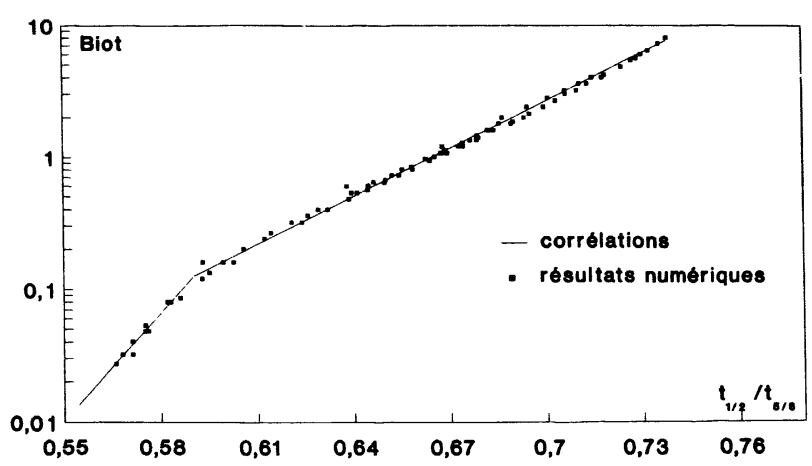

Fig. 8. - Fonction d'identification du nombre de Biot.

[Identification function of the Biot number.]

$a_{\text {apparent }}$, c'est-à-dire la diffusivité calculée à partir des données du thermogramme expérimental, $t_{1 / 3} / t_{5 / 6}$, $t_{1 / 2} / t_{5 / 6}, t_{2 / 3} / t_{5 / 6}$ et des corrélations de Degiovanni. La procédure d'identification se déroule ensuite de la façon suivante :

- le rapport $t_{1 / 2} / t_{5 / 6}$ relevé sur le thermogramme fournit une valeur estimée de $\rho_{2} C_{2}$, une valeur d'essai pour $a_{2}$ et par conséquent pour $h$ en vertu de la relation:

$$
h=\frac{a_{2} f\left(t_{1 / 2} / t_{5 / 6}\right)}{\rho_{2} C_{2} e_{2}}
$$

- sur la base de ces données, la température sur la face arrière de l'échantillon est calculée à chaque instant par la méthode des quadripôles. On obtient ainsi un thermogramme tel que :

$$
\begin{aligned}
& \left.\frac{t_{1 / 3}}{t_{5 / 6}}\right|_{\text {exp. }}=\left.\frac{t_{1 / 3}}{t_{5 / 6}}\right|_{\text {th. }} \\
& \left.\frac{t_{1 / 2}}{t_{5 / 6}}\right|_{\text {exp. }}=\left.\frac{t_{1 / 2}}{t_{5 / 6}}\right|_{\text {th. }} \\
& \left.\frac{t_{2 / 3}}{t_{5 / 6}}\right|_{\text {exp. }}=\left.\frac{t_{2 / 3}}{t_{5 / 6}}\right|_{\text {th. }}
\end{aligned}
$$

puisque le choix initial des données satisfait aux valeurs expérimentales de $t_{1 / 3} / t_{5 / 6}, t_{1 / 2} / t_{5 / 6}$ et $t_{2 / 3} / t_{5 / 6}$. L'identification entre les thermogrammes normalisés expérimental et théorique n'est pas suffisante pour déterminer la diffusivité de façon unique dans la mesure où tous les couples de valeurs $\left(a_{2}, h\right)$ qui satisfont à la relation (16) sont des solutions possibles au problème ainsi posé. La valeur de la diffusivité n'est accessible qu'à l'issue de l'identification des thermogrammes expérimental et théorique dimensionnés, ce qui est réalisé par la comparaison de deux temps, par exemple $t_{5 / 6}$ expérimental et la valeur de $t_{5 / 6}$ calculée à partir des données introduites dans la méthode des quadripôles. Les valeurs de $h$ et $a_{2}$ sont donc ajustées pour qu'il y ait concordance entre ces deux temps. 
3.4 VAlidation DE LA MÉTHOde. - La vérification de la procédure d'identification proposée a été réalisée : dans ce but, les thermogrammes issus de l'ensemble des cas traités avec le calcul bidimensionnel par volumes finis ont été dépouillés à l'aide de la procédure d'identification proposée plus haut. Le résultat obtenu a été comparé à la valeur de diffusivité initialement introduite dans le calcul 2D. Aucun écart notable n'a été relevé compte tenu des erreurs inhérentes à tout calcul numérique.

Par ailleurs, nous avons examiné l'influence d'une erreur sur l'estimation de la chaleur volumique. Pour cela, nous avons simulé le comportement d'un échantillon soumis à des flux différents du flux de référence pour étudier les conséquences de cette variation sur la précision avec laquelle on détermine $a_{2}$.

Dans les conditions très restrictives où nous opérons, on peut fixer à $20 \%$ le maximum d'écart que l'on puisse obtenir entre la valeur réelle de la chaleur volumique et sa valeur estimée. Pour les cas extrêmes $(-20 \%$ et $20 \%)$ l'erreur qui en résulte sur la valeur de la diffusivité de l'échantillon reste limitée puisqu'elle vaut respectivement $4 \%$ et $-2 \%$ (Fig. 9). Ces résultats sont satisfaisants et accréditent le fait qu'une simple estimation de $\rho_{2} C_{2}$ est suffisante pour une bonne détermination de la diffusivité.

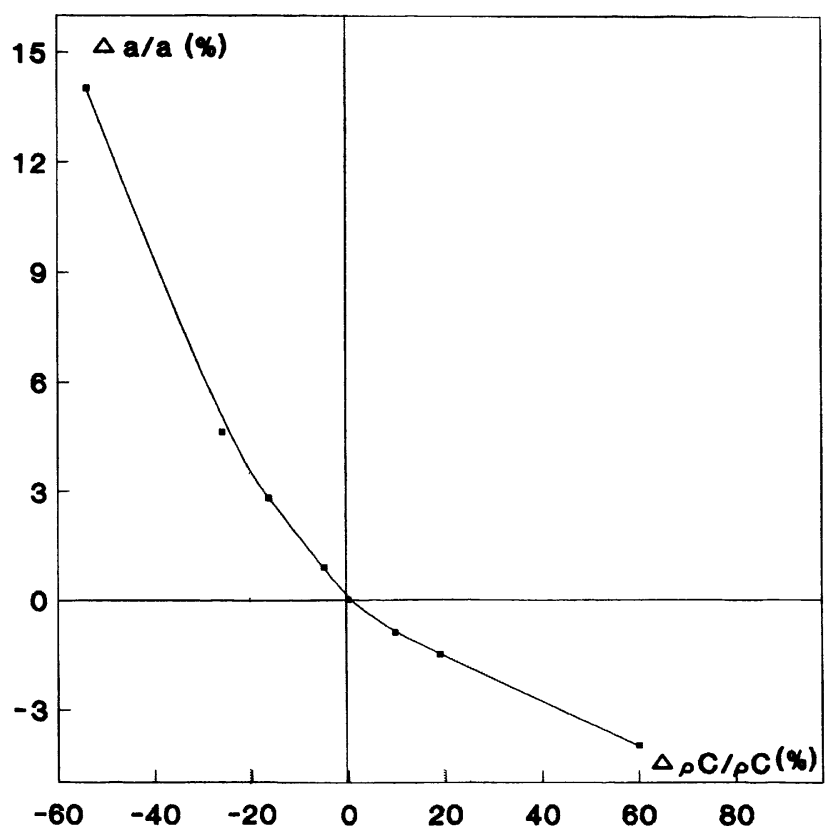

Fig. 9. - Conséquence d'une erreur d'évaluation de $\rho C$ sur l'identification de la diffusivité.

[Effect of the relative error of the $\rho C$ determination upon the diffusivity relative error.]

\section{Présentation des mesures.}

Bien que le montage expérimental soit essentiellement destiné à mesurer la diffusivité thermique de polymères solides ou fondus, la validation de la méthode, associée au processus d'identification, a été étendue à un ensemble plus large de produits. Nous avons réalisé un certain nombre de mesures sur :

- un échantillon de glycérine (température ambiante) ;

- un échantillon d'huile Rhodorsil (température ambiante) ;

- un échantillon de polyamide $6\left(25^{\circ} \mathrm{C}\right.$ à $\left.250^{\circ} \mathrm{C}\right)$.

$\mathrm{Ce}$ choix est directement lié au fait que pour l'ensemble de ces produits, nous disposions dans la littérature de valeurs de référence sur la gamme de température choisie. Les résultats présentés concernent les valeurs de chaleur volumique estimées (Tab. I) et les valeurs de diffusivité mesurées (Tab. II).

Tableau I. - Comparaison de nos mesures de diffusivité avec les valeurs de la littérature.

[Comparison with diffusivity measurements from the literature.]

\begin{tabular}{|l|c|c|c|c|}
\hline \multicolumn{1}{|c|}{ Produits } & $\begin{array}{c}\text { Température } \\
\left({ }^{\circ} \mathrm{C}\right)\end{array}$ & $\begin{array}{c}\rho C_{\text {estimé }} \\
\left(10^{6} \mathrm{~J} / \mathrm{m}^{3}{ }^{\circ} \mathrm{C}\right)\end{array}$ & $\begin{array}{c}\rho C_{\text {référence }} \\
\left(10^{6} \mathrm{~J} / \mathrm{m}^{3}{ }^{\circ} \mathrm{C}\right)\end{array}$ & $\begin{array}{c}\Delta \rho C / \rho C \\
(\%)\end{array}$ \\
\hline Glycérine & 25 & 3,04 & 3,02 & 0,7 \\
\hline Huile & 25 & 1,22 & 1,42 & 14,1 \\
Rhodorsil & & & & \\
\hline Polyamide 6 & 25 & 1,52 & 2,01 & 24,4 \\
& 50 & 1,68 & 2,09 & 19,1 \\
& 100 & 1,90 & 2,24 & 15,2 \\
& 190 & 2,39 & 2,64 & 9,5 \\
\hline
\end{tabular}

Tableau II. - Comparaison des estimations de chaleur volumique avec les valeurs de la littérature.

[Comparison with heat capacity values from the literature.]

\begin{tabular}{|l|c|c|c|c|}
\hline \multicolumn{1}{|c|}{ Produits } & $\begin{array}{c}\text { Température } \\
\left({ }^{\circ} \mathrm{C}\right)\end{array}$ & $\begin{array}{c}a_{\text {mesure }} \\
\left(10^{-7} \mathrm{~m}^{2} / \mathrm{s}\right)\end{array}$ & $\begin{array}{c}a_{\text {reference }} \\
\left(10^{-7} \mathrm{~m}^{2} / \mathrm{s}\right)\end{array}$ & $\begin{array}{c}\Delta a / a \\
(\%)\end{array}$ \\
\hline Glycérine & 25 & 0,99 & 0,95 & 4,2 \\
\hline Huile & 25 & 1,15 & 1,13 & 1,8 \\
Rhodorsil & & & & \\
\hline Polyamide 6 & 25 & 1,80 & 1,77 & 1,7 \\
& 50 & 1,63 & 1,67 & 2,4 \\
& 100 & 1,49 & 1,47 & 1,3 \\
& 190 & 1,05 & 1,07 & 1,9 \\
\hline
\end{tabular}

4.1 ESTIMATION DE CHALEURS VOLUMIQUES (Tab. I). - Les valeurs données comme référence pour la glycérine sont celles qui sont communément 
proposées dans la littérature [13]; pour l'huile Rhodorsyl, il s'agit des valeurs annoncées par le producteur (Rhône-Poulenc). Pour le polyamide 6, les valeurs de chaleur volumique, proposées comme base de comparaison, ne sont pas issues de mesures directes mais ont été calculées à partir des valeurs de conductivité et diffusivité thermique mesurées par Dietz [12].

Les écarts n'excèdent guère $20 \%$ ce qui, pour une simple estimation, reste très correct. Comme cela a déjà été mentionné au paragraphe 3.4 , l'influence d'une dispersion de cet ordre de grandeur pour la valeur de la chaleur volumique reste très limitée quant à l'erreur qu'elle peut engendrer sur la détermination de la diffusivité thermique, et la procédure d'identification proposée reste donc tout à fait justifiée.

4.2 Mesure DE Diffusivités THERMiQues (Tab. II). - Les valeurs de référence pour la glycérine et l'huile proviennent des mêmes sources que celles déjà précisées pour la chaleur volumique. Quant au polyamide 6, nous avons pris comme référence les mesures effectuées par Dietz [12] sur la même famille de polymères.

En règle générale, les écarts mis en évidence par rapport aux valeurs de référence restent inférieurs à $4 \%$.

\section{Conclusion.}

Nous pouvons estimer la précision avec laquelle sont obtenues les valeurs de diffusivité à $5 \%$, leur reproductibilité étant meilleure que $3 \%$. L'appareil est destiné à la mesure de diffusivité thermique de produits solides ou liquides jusqu'à $300^{\circ} \mathrm{C}$. Les premiers essais présentés montrent que l'ensemble du montage expérimental et du processus de dépouillement permettent de répondre de manière satisfaisante à cette ambition.

\section{Bibliographie}

[1] Gobbe C., Bazin M., Gounot J. and Dehay G., An apparatus for measuring polymer thermal conductivities at high pressure and temperature, J. Polym. Sci. 26 (1988) 857-864.

[2] Parker W. J., Jenkins R. J., Butler C. P. and Аввотт G. L., Method of determining thermal diffusivity, heat capacity and thermal conductivity, J. Appl. Phys. 32 (1961) 1679-1684.

[3] Degiovanni A. et Gery A., Etat actuel des techniques impulsionnelles appliquées à la détermination de la diffusivité thermique, Bulletin BNM (juil. 1976).

[4] Chen F. C., Poon Y. M. and Choy C. L., Thermal diffusivity of polymers by the flash method, Polymer 18 (1977) 129-135.

[5] Saulnier J. B., Dauron P. et Martinet J., Quelques aspects nouveaux de la méthode flash, Entropie 64 (1975) 44-51.

[6] Otter C. et Arles L., Mesure de la diffusivité thermique de l'étain liquide par la méthode du flash-laser. Conductivité thermique de l'étain liquide entre $1000^{\circ} \mathrm{C}$ et $1900^{\circ} \mathrm{C}$, Rev. Hautes Temp. Réfract. 15 (1978) 209-219.

[7] Kolenda Z. S., Nowakowski J. and Oblakowski R., Measurements of thermophysical properties of liquid electrolyte by modified heat pulse technique, Inst. J. Heat Mass Transfer 24 (1981) 891-894.

[8] Batsale J. C. et Degiovanni A., Extension de la méthode «flash» à deux cas particuliers : les matériaux anisotropes et les liquides, Journées SFT Limoges (mai 1988).

[9] Azizi S., Moyne C. et Degiovanni A., Approche expérimentale et théorique de la conductivité thermique des milieux poreux humides. 1-Expérimentation, Int. J. Heat Mass Tranfer 31 (1988) 2305-2317.
[10] Degiovanni A., Diffusivité et méthode flash, Rev. gen. Therm. 185 (1977) 420-441.

[11] Balageas D., Nouvelle méthode d'interprétation des thermogrammes pour la détermination de la diffusivité thermique par la méthode «flash", Rapport Technique ONERA n $12 / 3434$ (1981) 1-57.

[12] DieTz W., Contribution à l'étude thermodynamique des polymères à l'état fondu sous pression. Détermination de la conductivité thermique et de la diffusivité thermique, Thèse de Docteur Ingénieur, Université Paris VI (1976).

[13] Holman J. P., Heat Transfer (MacGraw-Hill) 1981.

[14] Lemesle-Gery A., Contribution à l'étude des mesures de température de surface par contacts séparés, en régimes permanent et transitoire, Thèse d'Etat, INSA, Université de Lyon I (avril 1980).

[15] Degiovanni A., Conduction dans un «mur » multicouche avec sources : extension de la notion de quadripôle, Int. J. Heat Mass Transfer 31 (1988) 553-557.

[16] Degiovanni A., Gery A., Laurent M., MacqueRON J. L. et SINICKI G., La méthode du signal bref appliquée à la détermination des caractéristiques thermocinétiques des solides, C. R. Acad. Sci. Paris 278 (1974) 49-52.

[17] Degiovanni A., Gery A., Laurent M. et Sinicki G., Détermination simultanée de la diffusivité, de la chaleur volumique et du comportement thermocinétique de matériaux solides, $C . R$. Acad. Sci., Paris 283 (1976) 167-170.

[18] KobAyASI K., Simultaneous measurement of thermal diffusivity and specific heat at high temperatures by a single rectangular pulse heating method, Int. J. Thermoph. 7 (1986) 181-195. 